\title{
Long-term evolution of patient-reported outcome measures in spinocerebellar ataxias
}

Heike Jacobi, MD*, Sophie Tezenas du Montcel, PhD, Peter Bauer, MD, Paola Giunti, PhD, Arron Cook, MBBS, Robyn Labrum, MD, Michael H. Parkinson, MBBS, Alexandra Durr, PhD, Alexis Brice, MD, Perrine Charles, MD, Cecilia Marelli, MD, Caterina Mariotti, MD, Lorenzo Nanetti, MD, Lidia Sarro, MD, Maria Rakowicz, PhD, Anna Sulek, PhD, Anna Sobanska, MD, Tanja SchmitzHübsch, MD, Ludger Schöls, MD, Holger Hengel, MD, Laszlo Baliko, MD, Bela Melegh, PhD, Alessandro Filla, MD, Antonella Antenora, MD, Jon Infante, MD, José Berciano, MD, Bart P. van de Warrenburg, PhD, Dagmar Timmann, MD, Sandra Szymanski, MD, Sylvia Boesch, MD, Wolfgang Nachbauer, MD, Jun-Suk Kang, MD, Massimo Pandolfo, MD, Jörg B. Schulz, MD, Audrey Tanguy Melac, MD, Alhassane Diallo, MD, Thomas Klockgether, MD.

Heike Jacobi, MD*, German Center for Neurodegenerative Diseases (DZNE), Bonn and Department of Neurology, Heidelberg University Hospital, Germany (heike.jacobi@ukb.unibonn.de)

Sophie Tezenas du Montcel, PhD, Sorbonne Universités, Université Pierre et Marie Curie (UPMC) Univ Paris 06, UMRS 1136, INSERM U 1136, Institut Pierre Louis d'Epidémiologie et de Santé Publique, F-75013, Paris, France and AP-HP, Biostatistics Unit, Groupe Hospitalier PitiéSalpêtrière, F-75013, Paris, France (sophie.tezenas@aphp.fr)

Peter Bauer, MD, Institute of Medical Genetics and Applied Genomics, University of Tübingen, Tübingen, and CENTOGENE AG, Rostock, Germany (Peter.Bauer@centogene.com)

Paola Giunti, PhD, Department of Molecular Neuroscience, UCL, Institute of Neurology, London, United Kingdom (p.giunti@ucl.ac.uk)

Arron Cook, MBBS, Department of Molecular Neuroscience, UCL, Institute of Neurology, London, United Kingdom (arroncook@doctors.org.uk)

Robyn Labrum, MD, Neurogenetic Laboratory, National Hospital of Neurology and Neurosurgery, UCL, London, United Kingdom (r.labrum@nhs.net)

Michael H. Parkinson, MBBS, Department of Molecular Neuroscience, UCL, Institute of Neurology, London, United Kingdom (m.parkinson@ucl.ac.uk)

Alexandra Durr, PhD, INSERM, U 1127, F-75013, Paris, France, CNRS, UMR 7225, F-75013, Paris, France, Sorbonne Universités, UPMC Univ Paris 06, UMRS_1127, F-75013, Paris, France, Institut du Cerveau et de la Moelle épinière, ICM, F-75013, Paris, France and APHP, Hôpital de la Pitié-Salpêtrière, Département de Génétique, F-75013, Paris, France (alexandra.durr@upmc.fr)

Alexis Brice, MD, INSERM, U 1127, F-75013, Paris, France, CNRS, UMR 7225, F-75013, Paris, France, Sorbonne Universités, UPMC Univ Paris 06, UMRS_1127, F-75013, Paris, France, Institut du Cerveau et de la Moelle épinière, ICM, F-75013, Paris, France and APHP, Hôpital de la PitiéSalpêtrière, Département de Génétique, F-75013, Paris, France (alexis.brice@upmc.fr)

Perrine Charles, MD, APHP, Hôpital de la Pitié-Salpêtrière, Département de Génétique, F-75013, Paris, France (perrine.charles@psl.aphp.fr)

Cecilia Marelli, MD, Service de Neurologie - CMRR, CHRU Gui de Chauliac, 80, av. A. Fliche, 34295 - Montpellier CEDEX 05, France (c-marelli@chu-montpellier.fr) 
Caterina Mariotti, MD, SOSD Genetics of Neurodegenerative and Metabolic Diseases, Fondazione-IRCCS Istituto Neurologico Carlo Besta, Milan, Italy (Caterina.Mariotti@istitutobesta.it)

Lorenzo Nanetti, MD, SOSD Genetics of Neurodegenerative and Metabolic Diseases, FondazioneIRCCS Istituto Neurologico Carlo Besta, Milan, Italy (Lorenzo.Nanetti@istituto-besta.it)

Lidia Sarro, MD, SOSD Genetics of Neurodegenerative and Metabolic Diseases, FondazioneIRCCS Istituto Neurologico Carlo Besta, Milan, Italy (lidia.sarro@istituto-besta.it)

Maria Rakowicz, PhD, Department of Clinical Neurophysiology, Institute of Psychiatry and Neurology, Warsaw, Poland (rakowicz@ipin.edu.pl)

Anna Sulek, PhD, Department of Genetics, Institute of Psychiatry and Neurology, Warsaw, Poland (suleka@ipin.edu.pl)

Anna Sobanska, MD, Department of Clinical Neurophysiology, Institute of Psychiatry and Neurology, Warsaw, Poland (asobanska@ipin.edu.pl)

Tanja Schmitz-Hübsch, MD, Charité Universitätsmedizin Berlin, NeuroCure Clinical Research Center, Berlin, Germany (tanja.schmitz-huebsch@charite.de)

Ludger Schöls, MD, Department of Neurodegeneration and Hertie-Institute for Clinical Brain Research, University of Tübingen and Deutsches Zentrum für Neurodegenerative Erkrankungen (DZNE), D-72076 Tübingen, Germany (Ludger.Schoels@uni-tuebingen.de)

Holger Hengel, MD, Department of Neurodegeneration and Hertie-Institute for Clinical Brain Research, University of Tübingen and Deutsches Zentrum für Neurodegenerative Erkrankungen (DZNE), D-72076 Tübingen, Germany (holger.hengel@uni-tuebingen.de)

Laszlo Baliko, MD, Department of Neurology, Zala County Hospital, H-8900 Zalaegerszeg, Zrinyi M. Str. 1., Hungary (balikolaszlo@freemail.hu)

Bela Melegh, PhD, Department of Medical Genetics, Medical School, and Szentagothai Research Center, University of Pécs, Pécs, Hungary (bela.melegh@aok.pte.hu)

Alessandro Filla, MD, Department of Neuroscience, and Reproductive and Odontostomatological Sciences, Federico II University Naples, Italy (afilla@unina.it)

Antonella Antenora, MD, Department of Neuroscience, and Reproductive and Odontostomatological Sciences, Federico II University Naples, Italy (antonella.antenora@virgilio.it)

Jon Infante, MD, Service of Neurology, University Hospital Marqués de Valdecilla (IDIVAL), University of Cantabria (UC) and Centro de Investigación Biomédica en Red de Enfermedades Neurodegenerativas (CIBERNED), Santander, Spain (jinfante@humv.es)

José Berciano, MD, Service of Neurology, University Hospital "Marqués de Valdecilla (IDIVAL)", "Centro de Investigación Biomédica en Red de Enfermedades Neurodegenerativas (CIBERNED)", University of Cantabria (UC), Santander, Spain (Jaberciano@humv.es)

Bart P. van de Warrenburg, PhD, Department of Neurology, Donders Institute for Brain, Cognition, and Behaviour, Radboud University Medical Center, PO Box 9101, 6500 HB Nijmegen, The Netherlands (b.vandewarrenburg@neuro.umcn.nl)

Dagmar Timmann, MD, Department of Neurology, University Clinic Essen, University of DuisburgEssen, Germany (dagmar.timmann@uni-essen.de)

Sandra Szymanski, MD, Department of Neurology, St. Josef Hospital, University Hospital of Bochum, Bochum, Germany (info-szymanski@t-online.de) 
Sylvia Boesch, MD, Department of Neurology, Medical University, Innsbruck, Innsbruck Austria (sylvia.boesch@i-med.ac.at)

Wolfgang Nachbauer, MD, Department of Neurology, Medical University, Innsbruck, Innsbruck, Austria (wolfgang.nachbauer@i-med.ac.at)

Jun-Suk Kang, MD, Department of Neurology, University of Frankfurt, Frankfurt/M, Germany (Junsuk.kang@em.uni-frankfurt.de)

Massimo Pandolfo, MD, Université Libre de Bruxelles (ULB), Neurology Service - ULB Hôpital Erasme, ULB Laboratory of Experimental Neurology, Brussels, Belgium (Massimo.pandolfo@ulb.ac.be)

Jörg B. Schulz, MD, Department of Neurology, RWTH Aachen University, Pauwelsstraße 30, 52074 Aachen and JARA-BRAIN Institute Molecular Neuroscience and Neuroimaging, Forschungszentrum Jülich $\mathrm{GmbH}$ and RWTH Aachen University, 52074 Aachen, Germany (jschulz@ukaachen.de)

Audrey Tanguy Melac, MD, AP-HP, Biostatistics Unit, Groupe Hospitalier Pitié-Salpêtrière, F75013, Paris, France (audrey.tanguy07@gmail.com)

Alhassane Diallo, MD, Sorbonne Universités, Université Pierre et Marie Curie (UPMC) Univ Paris 06, UMR S 1136, INSERM U 1136, Institut Pierre Louis d'Epidémiologie et de Santé Publique, F75013, Paris, France (alhassane.diallo@inserm.fr)

Thomas Klockgether, MD, Department of Neurology, University Hospital of Bonn, Bonn, Germany and German Center for Neurodgenerative Diseases (DZNE), Bonn, Germany (klockgether@uni$\underline{\text { bonn.de) }}$

*Corresponding author: Heike Jacobi, MD, Department of Neurology, Heidelberg University Hospital, Im Neuenheimer Feld 400, D-69120 Heidelberg, Germany, phone: +4962215636328; heike.jacobi@med.uni-heidelberg.de

Character count title: 83

Number of references: 28

Word count abstract: 249

Number of tables: 1

Word count paper: 3016

Number of figures: 3

Supplemental data

\section{Keywords:}

Spinocerebellar ataxia; Depression; Quality of life; ADL; Natural history studies; Trinucleotide repeat diseases 
Introduction: To study the long-term evolution of patient-reported outcome measures (PROMs) in the most common spinocerebellar ataxias (SCAs), we analyzed 8 year follow-up data of the EUROSCA natural history study, a cohort study of 526 patients with SCA1, SCA2, SCA3 and SCA6.

Methods: To assess functional capacity in daily living we used the functional assessment (part IV) of the Unified Huntington's disease rating scale (UHDRS-IV), for health-related quality of life the visual analogue scale of the EuroQol five dimensions questionnaire (EQ-5D VAS), and for depressive symptoms the patient health questionnaire (PHQ-9). Severity of ataxia was assessed using the Scale for the Assessment and Rating of Ataxia (SARA), neurological symptoms other than ataxia with the inventory of non-ataxia signs (INAS).

Results: UHDRS-IV [SCA1:-1.35(0.12); SCA2:-1.15(0.11); SCA3:-1.16(0.11); SCA6:-0.99(0.12)] and EQ-5D [SCA1:-2.88(0.72); SCA2:-1.97(0.49); SCA3:-2.06(0.55); SCA6:-1.03(0.57)] decreased linearly, whereas PHQ-9 increased [SCA1:0.15(0.04); SCA2:0.09(0.03); SCA3:0.06(0.04); SCA6:0.07(0.04)] during the observational period. Standard response means (SRMs) of UHDRSIV (0.473-0.707) and EQ-5D VAS (0.053-0.184) were lower than that of SARA (0.404-0.979). In SCA1, higher SARA scores [-0.0288(0.01), $p=0.0251]$, longer repeat expansions [-0.0622(0.02), $p=0.0002]$ and the presence of cognitive impairment at baseline [-0.5381(0.25), $p=0.0365]$ were associated with faster UHDRS-IV decline. In SCA3, higher INAS counts were associated with a faster UHDRS-IV decline [-0.05(0.02), $p=0.0212]$. In SCA1, PHQ-9 progression was faster in patients with cognitive impairment [0.14(0.07); $p=0.0396]$.

Conclusions: In the common SCAs, PROMs give complementary information to the information provided by neurological scales. This underlines the importance of PROMs as additional outcome measures in future interventional trials. 


\section{INTRODUCTION}

The spinocerebellar ataxias (SCAs) are autosomal dominantly inherited neurodegenerative diseases with prominent ataxia that lead to progressive disability during disease course. The SCAs are clinically and genetically heterogeneous and more than 40 genetically different types have been defined. The most common SCAs that together account for more than half of all affected families are SCA1, SCA2, SCA3 and SCA6. These subtypes are caused by translated CAG repeat expansions that encode elongated polyglutamine tracts within the proteins associated with each type. Patients with SCA1, SCA2 and SCA3 frequently present with additional neurological signs. Conversely, SCA6 is considered an almost purely cerebellar disorder.[1]

Recently, considerable progress has been made in characterizing progression of ataxia and additional neurological symptoms, but less is known about the progression of patient-reported outcome measures (PROMs). PROMs are increasingly recognized as valuable and essential for evaluating clinical care and treatment. Recently, the US-FDA developed a patient-reported outcome guidance related to the use of PROMs used to support drug approvals.[2] PROMs are collected through short, self-completed questionnaires and measure patient's subjective perception of health status. PROMs therefore provide important information beyond neurological scales.[3-4]

Previous cross-sectional studies reported that subjective health perception of SCA patients was impaired specifically due to functional impairment, pain and depressive symptoms.[5] As in other neurodegenerative diseases, the prevalence of depressive symptoms in SCA was higher than estimates from populations surveys.[6-8]

To study the evolution of PROMs including functional capacity in daily living, health-related quality of life and depressive symptoms as well as to identify factors that influence this evolution we analysed long term data of the EUROSCA natural history study, a multicentric longitudinal cohort study of 525 patients with either SCA1, SCA2, SCA3 or SCA6.[9-11] 


\section{METHODS}

\section{Study design}

In an 8-year longitudinal cohort study 525 patients with genetically confirmed SCA1, SCA2, SCA3 and SCA6 were recruited at 17 European ataxia referral centers which together form the EUROSCA Clinical Group. Assessments were done annually within 3 months around the specified time point for the first 3 years. Thereafter, participants entered an extension phase in which study visits were scheduled in connection with routine visits resulting in irregular intervals.

Assessments were done according to a written study protocol. The study was approved by the ethics committees of the participating centres and has therefore been performed in accordance with the ethical standards laid down in the 1964 Declaration of Helsinki and its later amendments. Written informed consent was obtained from all study participants. This study is registered with ClinicalTrials.gov, number NCT02440763.

\section{Procedures}

Functional capacity in daily living was assessed using the functional assessment (part IV) of the Unified Huntington's Disease Rating Scale (UHDRS)[12], a checklist of 25 common daily tasks yielding a sum score ranging from 0 to 25 by giving a score of 1 to each task the patient can perform.

To assess health-related quality of life we used the visual analogue scale (VAS) of the EuroQol five dimensions questionnaire (EQ-5D)[13] To calculate the patient is asked to mark the today's health status on a scale with endpoints of 0 and 100, indicating the "the worst" (0) and "the best health you can imagine" (100). To add qualitative information on health-related quality of life we additionally used the descriptive system of EQ-5D comprising five dimensions: mobility, self-care, usual activities, pain/discomfort and anxiety/depression. Each dimension has 5 levels: no problems, slight problems, moderate problems, severe problems and extreme problems. The patient is asked to indicate his/her health state in each of the five dimensions resulting in a 1-digit number that expresses the level selected for that dimension.

To assess the severity of depressive symptoms the depression scale of the Patient Health Questionnaire (PHQ-9) was used.[14] The PHQ-9 is a 9-item self-rating questionnaire that simply scores each of the nine DSM-IV criteria for depressive disorders. The severity of depression is calculated by assigning scores of $0,1,2$, and 3 , to the response categories "not at all", "several days", "more than half the days" and "nearly every day" respectively. The sum score ranges from 0 (absence of depression) to 27 (severe depression).

Severity of ataxia was assessed using the Scale for the Assessment and Rating of Ataxia (SARA) yielding a sum score that ranges from 0 to 40 with 0 indicating the absence and 40 the most severe degree of ataxia.[15] Neurological symptoms other than ataxia were assessed using the Inventory of Non-Ataxia Signs (INAS), yielding a sum score from 0 to 16 by counting the number of clinical signs (INAS count).[16] 


\section{Statistical analysis}

To investigate whether baseline characteristics of patients who had been followed only for the initial 3 years ( \pm 3 months) differed from those of patients who had been followed longer, we used t-tests for quantitative and chi-square tests for categorical variables.

For analysis of disease progression, a linear mixed model was applied with random effects on intercept and slope. The time variable was the year since inclusion. Because of the presence of a floor effect for a non-negligible part of the sample for the analysis of the PHQ-9 we used a curvilinear mixed model.[17] Curvilinear modeling takes into account that differences between two successive levels of the scale do not have a constant meaning and that the distribution of the scale greatly deviates from the normal distribution that is assumed in the linear mixed model. We used a spline link function for all SCAs. Linearity of the progression rate was tested via nested models (likelihood ratio test). Specifically, we tested for a linear and quadratic effect of time and choose the model that best fitted the data. To verify that dropouts did not affect the results, we used a patternmixture model to obtain an unbiased estimation of progression if the effect of disease duration was significant.[18] For comparison of the progression rates between the genotypes, the patternmixture model was adjusted on the genotype.

To identify factors that influenced progression of PROMs, the tested factor was added in interaction with the linear mixed model used for analysis, taking into account the missing data model of progression. The tested factors were the SARA, INAS, PHQ-9 and the presence of double vision, dysphagia, urinary dysfunction and cognitive impairment at baseline, sex, age at onset, disease duration and repeat length of the expanded and normal allele. Influence of these variables on the progression rates were tested via interactions between the given factor and the time variable. Independent factors that were significant in the univariate analysis were included in a multivariate model, including the interactions with back-ward selection. Estimates derived from the model are given as mean (SE).

To determine the sensitivity for change of the PROMs in comparison to the neurological scales, we calculated the standardized response mean (SRM), defined as mean score change per year divided by the standard deviation (SD) of the variation as effect size index. Values of $0.20,0.50$ and 0.80 were considered to represent small, moderate and large changes, respectively.[19] Statistical analyses were performed with SAS 9.3 software (SAS Institute, Cary, NC). All tests were two-sided. Test results were considered significant at the 0.05 level. Bonferroni correction was used for comparison of disease progression between genotypes. 


\section{RESULTS}

The study population consisted of 525 patients with SCA1, SCA2, SCA3 or SCA6. Analyses were performed in a subgroup of 462 patients who had at least one follow-up visit. 415 patients were seen for a follow-up visit after 1 year, 416 after 2 years, and 336 after 3 years. All following visits were done at irregular intervals. Flowcharts detailing the number of patients seen in each year, reasons for dropout and demographic data are given in the appendix.[11] While disease duration and SARA scores at baseline were similar in all genotypes, SCA6 patients had an older age at inclusion, older age of onset and lower number of non-ataxia signs. UHDRS-IV ( $p=0.8924)$, EQ5D-VAS $(p=0.2412)$ and PHQ-9 $(p=0.1394)$ did not differ between the genotypes at baseline. There was no difference in the frequencies of the use of anti-depressants between the genotypes $(p=0.8600)$.

We first compared patients who had been followed only for the initial 3 year period with those who had been followed longer. No group differences were observed in sex, age at inclusion, age at onset and repeat length of both, expanded and normal allele. However, in patients with a shorter follow-up, disease duration was longer (SCA1,SCA2) and SARA (SCA1,SCA2,SCA3) and INAS scores (SCA1,SCA3) were higher.[11] Additionally, UHDRS-IV scores [SCA1: 16.2(8.3) vs. 21.1(4.6), $p=0.0005$; SCA2: 16.3(7.7) vs. 19.5(6.6), $p=0.0079$; SCA3: 16.9(7.5) vs. 20.0(5.7), $p=0.0194$ ] as well as EQ-5D-VAS scores at baseline [SCA1: $51.9(23.4)$ vs. $66.5(18.9), p=0.0007$; SCA6: $56.6(20.3)$ vs. $67.0(17.8), p=0.0178$ ] were lower in those patients with a shorter follow-up, whereas PHQ-9 scores were higher in SCA1 patients with a shorter follow-up [8.6(6.7) vs. 5.0(5.6), $p=0.0035]$. Furthermore UHDRS-IV decrease was higher in SCA2 patients with a shorter follow-up $[-0.14(0.18)$ vs. $-0.07(0.08)]$.

The decline of UHDRS-IV scores best fitted with a linear model in all genotypes. As UHDRS-IV decline was faster in SCA2-patients with a maximum follow-up of 3 years, we applied a patternmixture model that allowed calculating an average annual decline of -1.35(0.12) in SCA1, 1.15(0.11) in SCA2, -1.16(0.11) in SCA3, and -0.99(0.12) in SCA6 (figure1). The decline did not differ between the genotypes $(p=0.3042)$. To identify factors that were independently associated with faster decline we applied multivariate modelling. In SCA1, higher SARA scores were associated with lower UHDRS-IV scores at baseline $[-0.7137(0.45)$ per additional SARA point, $p<0.001]$. Furthermore higher SARA scores [-0.0288(0.01), $p=0.0251]$, longer repeat expansions [$0.0622(0.02)$ per additional repeat unit, $p=0.0002$ ] and the presence of cognitive impairment at baseline $[-0.5381(0.25), p=0.0365]$ were associated with faster UHDRS-IV decline. In SCA3, higher INAS scores at baseline were associated with lower UHDRS-IV scores [-1.3588(0.26) per additional INAS point, $p<0.001]$ and a faster UHDRS-IV decline [-0.05(0.02), $p=0.0212]$, whereas we failed to identify factors associated with UHDRS-IV decline in SCA2 and SCA6 (table 1).

EQ-5D VAS decline best fitted with a linear model in all genotypes. The average decline was 2.88(0.72) per year in SCA1, -1.97(0.49) in SCA2, -2.06(0.55) in SCA3, and -1.03(0.57) in SCA6 (figure2). The decline did not differ between the genotypes $(p=0.2993)$. In SCA1, we found that 
higher SARA scores [-0.7374(0.18), $\mathrm{p}<0.001]$ and the presence of dysphagia [-8.6842(3.37), $p=0.0113$ ] at baseline were associated with lower EQ-5D-VAS scores. Furthermore the presence of dysphagia at baseline attenuated the decline to 2.46(0.99) $(p=0.0157)$ whereas longer repeat expansions [-0.2264(0.09), $p=0.0176]$ were associated with faster decline. We failed to identify factors associated with EQ-5D-VAS decline in SCA2, SCA3 and SCA6 (table1).

With regard to the five dimensions of subjective health perception impairment of self-care increased in all genotypes with an average of 0.39(0.07) per year in SCA1, 0.25(0.05) in SCA2, $0.41(0.08)$ in SCA3, and $0.26(0.07)$ in SCA6 as well as the subjective impairment of mobility with an average of $0.44(0.13)$ in SCA1, 0.24(0.06) in SCA3 and 0.22(0.07) in SCA6 per year. In SCA2 and SCA3 patients furthermore reported an aggravation of problems in usual activities $(0.20(0.04)$; $0.17(0.04)$ ) whereas subjective problems due to depression and anxiety increased only in SCA1 with an average of $0.10(0.04)$ and in SCA2 with $0.10(0.03)$.

The evolution of PHQ-9 sum score was described with a curvilinear mixed model in all genotypes. The progression was similar between patients who have been followed only for the initial 3 year period and those who have been followed longer in SCA1 [-0.050(0.15); $p=0.7332]$, SCA3 [$0.005(0.10) ; p=0.9616]$ and SCA6 [-0.020(0.11); $p=0.8311]$. However, in SCA2 the patients with a shorter follow-up had a faster progression of PHQ-9 [0.37(0.08); $p<0.00001]$. PHQ-9 increased significantly in SCA1 [0.15(0.04); $p=0.0009$ ] and SCA2 [0.09(0.03); $p=0.0010]$, but this increase was not significant in SCA3 [0.06(0.04); $p=0.0872$ ] and SCA6 [0.07 (0.04); $p=0.0905]$. The evolution of PHQ-9 did not differ between the genotypes ( $p>0.0083$ ).

To identify factors that were independently associated with faster PHQ-9 progression we applied multivariate modeling (table 1). In SCA1 patients, progression was faster for patients with cognitive impairment [0.14(0.07); $p=0.0396]$. In SCA2, men showed significantly lower PHQ-9 scores [$0.46(0.23) ; p=0.0492]$ while patients suffering from dysphagia [0.29(0.14); $p=0.0301]$, double vision [0.37(0.15); $p=0.0159]$ and urinary dysfunction [0.52(0.17); $p=0.0019]$ showed higher scores. Evolution of PHQ-9 was faster for patients with a shorter follow-up [0.37(0.08); $p=0.0001]$ and for men [0.11(0.05); $p=0.04211]$ while it decreased with the presence of urinary dysfunction [$0.11(0.05) ; p=0.04260]$. We did not identify factors influencing the evolution of PHQ-9 in SCA3 and SCA6.

SRMs reached the criterion of moderate change for UHDRS-IV [SRM 0.473-0.707]. They were in the same range as the SRMs of INAS count changes [0.360-0.662], but smaller than those of SARA changes [0.404-0.979]. In contrast, SRMs for EQ-5D VAS were negligible [SRM 0.0530.184] (Appendix). We were not able to calculate the SRMs of PHQ-9 changes due to the curvilinear model. 


\section{DISCUSSION}

Our study provides a comprehensive account of the long-term evolution of PROMs including functional capacity in daily living, health-related quality of life and depressive symptoms in the most common SCA disorders that steadily change over the entire observational period. As there was no validated measure of functional capacity specific for ataxic patients available at the start of our study, we decided to use UHDRS-IV. This scale was developed for assessment of patients with Huntington's disease.[12] However, the use seemed appropriate as patients with ataxia and Huntington`s disease face qualitatively similar functional limitations in daily life. In addition, in the SARA validation trials, UHDRS-IV was strongly correlated with SARA.[15] For assessment of subjective health perception we applied EQ-5. As other visual analogue scales, EQ-5D VAS has the inherent problem of the end-of-scale bias, i.e. that the extreme ends of the scale are less likely to be used. On the other hand, EQ-5D is easy to use, available in validated translations in different languages and provides quantitative and qualitative information about patient's perception of health state. Depression is known to have a major impact on subjective health perception in various neurodegenerative diseases.[5, 20] In this study, we used PHQ-9 to assess severity of depression. A previous study in SCA patients proved that PHQ-9 is a valid instrument to detect clinical relevant depression.[5] PHQ-9 is a brief self-rating questionnaire that is known for its excellent feasibility, reliability, validity and sensitivity but lower specificity and positive predictive value.[21-23] Overall it has been shown to be a valid measure of depression in different cultures and translations.[24] The use of PHQ-9 in ataxia patients is complicated by the fact that one of the 9 items is related to slowness of movements and speech which may be due to ataxia rather than reflecting depressive mood.

Strengths of our study include the large number of patients and the long observational period of up to 8 years. However, there was a considerable dropout rate. In our previous analysis of the evolution of ataxia and neurological symptoms in this cohort, we found that the dropout rate was particularly high in those who had a longer disease duration (SCA1,SCA2) and greater disease severity at baseline (SCA1,SCA2,SCA3). Correspondingly, in SCA1, SCA2 and SCA3, baseline UHDRS-IV scores, and, in SCA1 and SCA6, EQ-5D-VAS scores were lower in patients that were followed only for the initial 3 years. To account for the dropout and estimate PROM evolution in an unbiased way, we applied a pattern-mixture model approach. Pattern-mixture models consider missing data as a random process which means that the probability of having missing data depends on the other observed information. The average evolution is thus estimated as the

weighted mean of the evolution in the group with a follow-up of maximally 3 years and the evolution in the group with extended follow-up.

In the last years PROMs are increasingly considered as relevant outcome measures as they directly assess patient's perception of health status and may better reflect the impact of the disease on everyday life than clinical scales. The most important finding of our analysis is that all three measures, functional capacity, health-related quality of life and mood deteriorated over the entire observation period in all genotypes. The 2-year longitudinal CRC-SCA study did not find an 
increase of PHQ-9 in SCA1, SCA2, SCA3 and SCA6.[7] This is in line with previous studies in Huntington's disease that did not find an increase of depression at any stage of disease.[25-26] The reason for the discrepancy to our findings may be the shorter observation time of the other studies. Although we observed steady changes of all PROMs, the effect sizes, which could be determined for methodological reasons only for UHDRS-IV and EQ-5D, were considerably smaller than that of SARA. The use of UHDRS-IV or EQ-5D as a primary outcome measure in a clinical trial would thus be associated with much larger patient numbers. Our previous analysis of the evolution of ataxia severity in this cohort revealed that progression was fastest in SCA1, intermediate in SCA2 and SCA3, and slowest in SCA6.[11] In contrast, the present analysis did not show significant differences of the change rate of the PROMs between the genotypes. This may be simply due to the larger variability of the PROMs resulting in a lack of power, but may also be due to the fact that PROMs reflect different aspects of the disease than clinical scales, such as SARA. In SCA1, SARA was a determinant of the UHDRS-IV and EQ-5D VAS baseline and the UHDRS-IV rate of decline. Furthermore, length of the expanded allele which is a major determinant of SARA progression in SCA1, was associated with faster decline of UHDRS-IV and EQ-5D VAS in SCA1. On the other hand, we did not find a major influence of SARA on any PROM in SCA2, SCA3 and SCA6. This is in line with studies in other neurodegenerative disorders that have shown that patient's subjective health-perception is only partly related to clinical assessment of diseaserelated impairment, but may be also influenced by other factors such as emotional well-being and other comorbidities.[27] INAS, which is a measure of extracerebellar involvement, affected both baseline UHDRS-IV and UHDRS-IV decline in SCA3. These observations underline the view that, although severity of neurological involvement, as measured by SARA and INAS, has an impact on functional capacity and subjective health perception, it only explains a part of the variablity of these PROMs. In SCA1, functional capacity appears to be strongly related to ataxia, whereas in SCA3 which is the prototype of a SCA with a highly pleomorphic phenotype neurological symptoms other than ataxia influence functional capacity.[28]

Depressive symptoms were mainly influenced by non-motor features, such as cognitive impairment (SCA1), as well as urinary dysfunction and male sex (SCA2). Similarly, the authors of the CRCSCA study concluded that depressive symptoms are not simply the consequence of motor disability.[7] In our study cognitive impairment was solely assessed according to the clinical impression of the examiner. This impression may be confounded for example by the occurrence of depressive pseudo-dementia, however prevalence of relevant depressive syndrome was not high in this study. However, the influence of cognitive impairment on the evolution of UHDRS-IV and PHQ-9 in SCA1 has to be interpreted with caution.

Our study gives useful information on the long-term evolution of PROMs in the common SCAs. The decline of functional capacity and quality of life along with the increase of depressive symptoms were partly related to deterioration of motor abilities, but were not fully explained by them. In the common SCAs, PROMs thus give complementary information to the information provided by 
clinical scales. This underlines the importance of PROMs as additional outcome measures in future interventional trials.

\section{ACKNOWLEDGEMENT}

This study was funded within the framework of EU FP6 (EUROSCA). Additional funds came from the German Ministry of Education and Research (GeneMove), Polish Ministry of Scientific Research and Information Technology, Hungarian funding agency (NKFIH 119540) and from the European Community's Seventh Framework Programme (FP7/2007-2013) under grant agreement $\mathrm{n}^{\circ}$ 2012-305121 "Integrated European-omics research project for diagnosis and therapy in rare neuromuscular and neurodegenerative diseases (NEUROMICS)". The sponsors had no role in the study design, data collection, data analysis, data interpretation, or writing of the report. The corresponding author had full access to all the data in the study and had final responsibility for the decision to submit for publication.

We are grateful to Drs. Klopstock and Petersen (Department of Neurology, University of Munich, Munich, Germany) and Dr. Delnooz (Department of Neurology, Radboud University Nijmegen Medical Center, Nijmegen, The Netherlands) for contribution of patients and help in patient assessment. Paola Giunti and Michael Parkinson work at University College London Hospitals/University College London, which receives a proportion of funding from the Department of Health's National Institute for Health Research Biomedical Research Centres funding scheme, and receives support from the Dementias and Neurodegenerative Diseases Research Network (DeNDRoN). 


\section{FINANCIAL DISCLOSURES AND CONFLICTS OF INTEREST}

H. Jacobi reports no disclosures.

S. Tezenas du Montcel reports no disclosures.

P. Bauer is executive board member at Centogene AG, Rostock, GER. He holds stock options in the company. He received speaker honoraria from Actelion Pharmaceuticals, Aschwil, $\mathrm{CH}$. His research work was partly funded by EC grants (2012-305121).

P. Giunti received research support from Pfizer, Reata, Acetalion unrelated to the topic of this paper. She received funding for a trip to attend a meeting organised by Acetalion.

A. Cook reports no disclosures.

R. Labrum reports no disclosures.

M. Parkinson reports no disclosures.

A. Durr holds a patent "Anaplerotic therapy of Huntington disease and other polyglutamine diseases", BIO06353 Mochel/Durr (EP 06291873.5, 12/4/2006)".

A. Brice serves on the scientific advisory board for FWO (Research Foundation Flanders), ERC (European Research Council), as an editorial board member for the journals Neurology and Clinical Neuroscience, Brain, Neurodegenerative Diseases, The Cerebellum, Neurogenetics. He received grants from the public French Research Agency (ANR), EU, France Parkinson Association, RDS (Roger de Spoelberch Foundation), FDF (Fondation de France) and FRM (Fondation pour la recherche médicale).

P. Charles reports no disclosures.

C. Marelli receveid financial support for travel expenses and congres participation from Acetlion Pharmaceutical (unrelated to this study).

C. Mariotti received funding for an educational seminar from Roche Pharmaceuticals and research support from the Italian Ministry of Health (Grant \# RF-2011-02347420), CHDI Foundation and Telethon UILDM (Grant \# GUP15009).

L. Nanetti, received research support from the Italian Ministry of Health (Grant \# GR-201302357821).

L. Sarro received research support from the Italian Ministry of Health (Grant \# RF-2011-02347420).

M. Rakowicz received research support from the Polish Ministry of Science and Higher Education grant 674N-RISCA/2010-2014 and Clinical TeleNeuroforma; grant IS-2/230/NCBR/2015 2016 from the National Science Center Poland and a Speaker's honorarium from the TERMEDIA Ltd.

A. Sulek reports no disclosures.

A. Sobanska part in Clinical TeleNeuroforma project sponsored by grant IS-2/230/NCBR/2015.

T. Schmitz-Hübsch reports no conflicts of interest. She was funded by EU and Bundesminsterium für Wirtschaft und Energie.

L. Schöls reports no disclosures relevant to this manuscript. L. Schöls received grants from EU FP7 and the Deutsche Forschungsgemeinschaft (DFG) during conduct of this study outside the submitted work.

H. Hengel reports no disclosures.

L. Baliko reports no disclosures. 
B. Melegh reports no disclosures.

A. Filla reports no disclosures.

A. Antenora reports no disclosures.

J. Infante received research support from CIBERNED and honoraria as speaker and financing the attendance at meetings from Zambon and Abbvie.

J. Berciano reports no disclosures.

B. van de Warrenburg is receiving or has received research grants from Hersenstichting, Gossweiler Foundation, Radboud university medical centre, BBMRI-NL, ZonMW, and Bioblast Pharma.

D. Timmann was funded by the German Research Foundation (DFG), Mercator Research Center Ruhr (MERCUR) and the German Heredoataxia Foundation (DHAG).

S. Szymanski reports no disclosures.

S. Boesch served on the advisory boards for Gruenenthal and Abbvie, received honoraria from Ipsen, Allergan, Abbvie, Novartis, Gruenenthal and Reata and the following grants: European Friedreich Ataxia Consortium for Translational Studies (EFACTS), FP7 Health (HEALTH-F2-2010242193), E-Rare-3 "Clinical research for new therapeutic uses of already existing molecules (repurposing) in rare diseases"(E-Rare-3JTC2016).

W. Nachbauer reports no disclosures.

J.-S. Kang served as consultant and received honoraria and funding for congress fees and travel expenses from Merz Pharma $\mathrm{GmbH}$, received honoraria from Medtronic $\mathrm{GmbH}$ and funding for congress fees from Ipsen Pharma.

M. Pandolfo served on the scientific advisory board for Voyager Therapeutics and Biomarin and as associate editor for Neurology Genetics, receives royalties from Athena Diagnostics and a research grant to perform studies in cellular and animal models of Friedreich ataxia from Biomarin.

J. Schulz reports grants from BMBF (GeNeMove) grants from EU (EUROSCA) during the conduct of the study; independent of the work presented here he receives grants from the EU (HEALTH-F22010- 242193). J. Schulz serves on scientific advisory boards for Lundbeck Inc., TEVA, Novartis, ForwardPharma and Lilly; he has received funding for travel and speaker honoraria from GlaxoSmithKline, Merz Pharmaceuticals, Medical Tribune, Lundbeck Inc., Pfizer Inc, Boehringer, Bayer. He serves as Editor-in-Chief of the Journal of Neurochemistry and Associate Editor for eNeuro.

A.Tanguy worked for Lundbeck SAS and AbbVie Inc outside of the submitted work.

A. Diallo reports no disclosures.

T. Klockgether reports grants from EU FP7 Neuromics, during the conduct of the study; personal fees from Biohaven, personal fees from ICON Clinical Research, personal fees from Atheneum, outside the submitted work. 


\section{REFERENCES:}

[1] Paulson HL, Shakkottai VG, Clark HB, Orr HT. Polyglutamine spinocerebellar ataxias from genes to potential treatments. Nat Rev Neurosci. 20170ct;18(10):613-626.

[2] U.S. Department of Health and Human Services Food and Drug Administration; Center for Drug Evaluation and Research (CDER); Center for Biologics Evaluation and Research (CBER); Center for Devices and Radiological Health (CDRH). Guidance for Industry Patient-Reported Outcome Measures:Use in Medical Product Development to Support $\begin{array}{lll}\text { Labeling } & \text { Claims. } & 2009 .\end{array}$ https://www.fda.gov/downloads/drugs/guidances/ucm193282.pdf

[3] Recinos PF, Dunphy CJ, Thompson N, Schuschu J, Urchek JL 3rd, Katzan IL. Patient Satisfaction with Collection of Patient-Reported Outcome Measures in Routine Care. Adv Ther. 2017Feb;34(2):452-465.

[4] Bryan S, Davis J, Broesch J, Doyle-Waters MM, Lewis S, McGrail K, et al. Choosing your partner for the PROM: a review of evidence on patient-reported outcome measures for use in primary and community care. Healthc Policy. 2014Nov;10(2):38-51.

[5] Schmitz-Hübsch T, Coudert M, Giunti P, Globas C, Baliko L, Fancellu R, et al. Self-rated health status in spinocerebellar ataxia--results from a European multicenter study. Mov Disord. 2010Apr15;25(5):587-95.

[6] Schmitz-Hübsch T, Coudert M, Tezenas du Montcel S, Giunti P, Labrum R, Dürr A et al. Depression comorbidity in spinocerebellar ataxia. Mov Disord. 2011Apr;26(5):870-6.

[7] Lo RY, Figueroa KP, Pulst SM, Perlman S, Wilmot G, Gomez C et al. Depression and clinical progression in spinocerebellar ataxias. Parkinsonism Relat Disord. 2016Jan;22:8792.

[8] Cecchin CR, Pires AP, Rieder CR, Monte TL, Silveira I, Carvalho T et al. Depressive symptoms in Machado-Joseph disease (SCA3) patients and their relatives. Community Genet. 2007;10(1):19-26.

[9] Schmitz-Hübsch T, Coudert M, Bauer P, Giunti P, Globas C, Baliko L et al. Spinocerebellar ataxia types 1, 2, 3, and 6: disease severity and nonataxia symptoms. Neurology. 2008Sep 23;71(13):982-9.

[10] Jacobi H, Bauer P, Giunti P, Labrum R, Sweeney MG, Charles P et al. The natural history of spinocerebellar ataxia type 1, 2, 3, and 6: a 2-year follow-up study. Neurology. 2011Sep13;77(11):1035-41.

[11] Jacobi H, du Montcel ST, Bauer P, Giunti P, Cook A, Labrum R et al. Long-term disease progression in spinocerebellar ataxia types 1,2, 3, and 6: a longitudinal cohort study. Lancet Neurol. 2015Nov;14(11):1101-08.

[12] Unified Huntington's Disease Rating Scale: reliability and consistency. Huntington Study Group. Mov Disord. 1996Mar;11(2):136-42. 
[13] EuroQol Group. EuroQol--a new facility for the measurement of health-related quality of life. Health Policy. 1990 Dec;16(3):199-208.

[14] Spitzer RL, Kroenke K, Williams JB. Validation and utility of a self-report version of PRIMEMD: the PHQ primary care study. JAMA 1999;282:1737-44.

[15] Schmitz-Hübsch T, du Montcel ST, Baliko L, Berciano J, Boesch S, Depondt C et al. Scale for the assessment and rating of ataxia: development of a new clinical scale. Neurology. 2006Jun13;66(11):1717-20.

[16] Jacobi H, Rakowicz M, Rola R, Fancellu R, Mariotti C, Charles P et al. Inventory of NonAtaxia Signs (INAS): validation of a new clinical assessment instrument. Cerebellum. 2013Jun;12(3):418-28.

[17] Proust C, Jacqmin-Gadda H, Taylor JM, Ganiayre J, Commenges D. A nonlinear model with latent process for cognitive evolution using multivariate longitudinal data. Biometrics. 2006Dec;62(4):1014-24.

[18] Verbeke G, Molenberghs G. Pattern-mixture models. Linear mixed models for longitudinal data. New York: Springer, 2000:275-93.

[19] Kazis LE, Anderson JJ, Meenan RF. Effect sizes for interpreting changes in health status. Med Care 1989;27(3 suppl):178-189.

[20] Simuni T, Sethi K. Nonmotor manifestations of Parkinson's disease. Ann Neurol. 2008Dec;64 Suppl 2:S65-80.

[21] Kroenke K, Spitzer RL, Williams JB. The PHQ-9: validity of a brief depression severity measure. J Gen Intern Med. 2001Sep;16(9):606-13.

[22] Furukawa TA: Assessment of mood: guides for clinicians. J Psychosom Res. 2010,68:581589.

[23] Inoue T, Tanaka T, Nakagawa S, Nakato $\mathrm{Y}$, Kameyama R, Boku $\mathrm{S}$ et al. Utility and limitations of PHQ-9 in a clinic specializing in psychiatric care. BMC Psychiatry. 2012Jul3;12:73.

[24] Gilbody S, Richards D, Brealey S, Hewitt C. Screening for depression in medical settings with the Patient Health Questionnaire (PHQ): a diagnostic meta-analysis. J Gen Intern Med. 2007Nov;22(11):1596-602.

[25] Thompson JC, Harris J, Sollom AC, Stopford CL, Howard E, Snowden JS et al. Longitudinal evaluation of neuropsychiatric symptoms in Huntington's disease. J Neuropsychiatry Clin Neurosci. 2012Winter;24(1):53-60.

[26] van Duijn E, Reedeker N, Giltay EJ, Eindhoven D, Roos RA, van der Mast RC. Course of irritability, depression and apathy in Huntington's disease in relation to motor symptoms during a two-year follow-up period. Neurodegener Dis. 2014;13(1):9-16.

[27] Ho AK, Gilbert AS, Mason SL, Goodman AO, Barker RA. Health-related quality of life in Huntington's disease: Which factors matter most? Mov Disord. 2009Mar15;24(4):574-8. 
[28] Rosenberg RN. Machado-Joseph disease: an autosomal dominant motor system degeneration. Mov Disord. 1992;7(3):193-203. 